\title{
SMOOTH GROUP ACTIONS ON SPHERE BUNDLES OVER SPHERES AND ON BRIESKORN MANIFOLDS
}

\author{
MIKIYA MASUDA ${ }^{1}$
}

\begin{abstract}
This paper solves one of the problems in [2] proposed by $M$. Davis. Our result asserts that natural smooth actions on certain sphere bundles over spheres are distinct from those on certain Brieskorn manifolds. Besides this an alternative proof to a result of Davis is given which seems rather more direct than his proof.
\end{abstract}

0. Introduction. Let $\Lambda$ stand for either the quaternion or Cayley numbers (denoted by $H$ or $O$ respectively). For each pair of integers $(h, k)$, let $E_{h, k}(\Lambda)$ be the manifold formed by identifying two copies of $\Lambda \times \Lambda$ via the diffeomorphism of $(\Lambda-\{0\})$,

$$
(u, v) \rightarrow\left(u^{\prime}, v^{\prime}\right)=\left(\frac{u}{|u|^{2}}, \frac{u^{h} v u^{k}}{|u|^{h+k}}\right) .
$$

The natural projection $E_{h, k}(\Lambda) \rightarrow \Lambda P^{1}$ induced by the projection of $\Lambda \times \Lambda$ to the first factor gives $E_{h, k}(\Lambda)$ the structure of a vector bundle over $\Lambda P^{1}$ where $\Lambda P^{1}$ is the $\Lambda$-projective line.

Let $S_{h, k}(\Lambda)$ be the total space of the unit sphere bundle of $E_{h, k}(\Lambda)$. Since the group of $R$-algebra automorphisms of $\Lambda$, written $G^{\Lambda}$, naturally acts on $S_{h, k}(\Lambda)$, we get a series of $G^{\Lambda}$-manifolds $S_{h, k}(\Lambda)$. Here, as is well known, $G^{\Lambda}=\operatorname{SO}(3)$ if $\Lambda=H$ and $G^{\Lambda}=G_{2}$ (the exceptional compact Lie group of rank 2) if $\Lambda=O$.

On the other hand, for each positive integer $m$, there is a Brieskorn manifold $\Sigma_{m}^{2 d+1}$ defined as

$$
\Sigma_{m}^{2 d+1}=\left\{\left(w, z_{0}, \ldots, z_{d}\right) \in C^{d+2} \mid w^{m}+z_{0}^{2}+\cdots+z_{d}^{2}=0\right\} \cap S^{2 d+3}
$$

where $d=\operatorname{dim}_{R} \Lambda-1$. This supports an $O(d)$-action which operates linearly on the last $d$-coordinates. Since $G^{\Lambda}$ can be naturally regarded as a subgroup of $O(d)$, we get another series of $G^{\Lambda}$-manifolds $\Sigma_{m}^{2 d+1}$.

M. Davis observed in [2] that their orbit spaces have the same structure if and only if $h+k= \pm m$ and posed the following problem (Problem 6 on p. 83 of [2]).

Problem. Is $\Sigma_{m}^{2 d+1} G^{\Lambda}$-diffeomorphic to $S_{h, k}(\Lambda)$ with $h+k= \pm m$ ?

The following theorem settles the problem.

THEOREM A. $\Sigma_{m}^{2 d+1}$ is $G^{\Lambda}$-diffeomorphic to $S_{h, k}(\Lambda)$ if and only if $m=1$ and $\{h, k\}=\{ \pm 1,0\}$ as a set.

REMARK. When $m=1$ and $\{h, k\}=\{ \pm 1,0\}$, it is easily checked that they are isomorphic to a linear action on $S^{2 d+1}$.

Received by the editors May 31, 1983.

1980 Mathematics Subject Classification. Primary 57S15, 57S25.

Key words and phrases. Smooth actions.

${ }^{1}$ The author is partially supported by the Sakkokai Foundation

(C) 1984 American Mathematical Society $0002-9939 / 84 \$ 1.00+\$ .25$ per page 
In this paper we have one more aim.

Let $\tilde{C}_{7}(\mathrm{SO}(2))$ (the notation is due to [2]) be the set of equivariant diffeomorphism classes of all oriented homotopy 7 -spheres with a semifree $S^{\mathbf{1}}$-action whose fixed point set is diffeomorphic to the standard 3-sphere. It is known that the equivariant connected sum around a fixed point gives it a group structure and that this group is infinite cyclic (see $\S 2$ ).

As is easily checked, $S_{h, k}(H)$ with the above semifree $S^{1}$-action belongs to $\tilde{C}_{7}(\mathrm{SO}(2))$ if and only if $h+k= \pm 1$. Under these circumstances there is the following interesting result obtained by Davis.

THEOREM B (PROPOSITION 7.15 OF $[2]) . S_{2,-1}(H)$ with the above semifree $S^{1}$-action is exactly a generator of $\tilde{C}_{7}(\mathrm{SO}(2))$.

This theorem is used in [4] to show the existence of an orientation preserving smooth involution on every homotopy $C P^{3}$.

Our additional aim is to give an alternative proof to Theorem B which seems rather direct.

This paper is organized as follows. In $\S 1$ Theorem $A$ is verified. In $\S 2$ we recall the Montgomery-Yang correspondence and state a known result concerning it. Making use of it, an alternative proof of Theorem B is given in $\S 3$.

Throughout this paper we always remain in the $C^{\infty}$-category.

1. Proof of Theorem A. We first treat the case where $\Lambda=H$. In this case, $G^{\Lambda}=\mathrm{SO}(3)$ and the subgroup of $G$ fixing the complex numbers of $H$ is a circle subgroup. Hereafter, on $S_{h, k}(H)$ we always consider the $S^{1}$-action given by the restriction to this circle subgroup of $\mathrm{SO}(3)$, and on $\Sigma_{m}^{7}$ the restricted action of $S^{1}(=\mathrm{SO}(2))$ which rotates the last two coordinates. To verify the theorem in the case where $\Lambda=H$, it is sufficient to show that $S_{h, k}(H)(h+k= \pm m)$ and $\Sigma_{m}^{7}$ are not equivariantly diffeomorphic as the $S^{1}$-manifolds because any of the circle subgroups of $\mathrm{SO}(3)$ are conjugate to each other by the maximal torus theorem.

We notice that these $S^{1}$-actions are semifree and that their fixed point sets are both diffeomorphic to the 3-dimensional lens space of type $(m, 1)$. The normal bundle $\xi$ of each $S^{1}$-fixed point set has a unique complex structure induced from the $S^{1}$-action such that $g_{*} w=g w$ for $g \in S^{1} \subset C, w \in \xi$. Hereafter we always understand that a normal bundle of an $S^{1}$-fixed point set is endowed with the complex structure in this way.

We shall investigate their Chern classes. The following lemma is not difficult to prove and the proof is left to the reader.

LEMMA 1.1. The complex normal bundle of the $S^{1}$-fixed point set in $\Sigma_{m}^{7}$ is trivial, and hence it has the trivial Chern classes.

For $S_{h, k}(H)$, however, it is not necessarily trivial as shown in the following.

Let $D_{h, k}(H)$ be the total space of the unit disk bundle of $E_{h, k}(H)$. Clearly this also admits a semifree $S^{1}$-action naturally extending the one on $S_{h, k}(H)$. We denote by $F$ the fixed point set of $S^{1}$ on $D_{n, k}(H)$ and by $S$, which is a 2-sphere, the subset of $F$ defined by $v=0, v^{\prime}=0$ (see Introduction).

Let $\nu$ be the complex normal bundle of $F$ and let $\left.\nu\right|_{S},\left.\nu\right|_{\partial F}$ be its restriction to $S, \partial F$ (the boundary of $F$ ) respectively. 
LEMMA 1.2. The first Chern class $c_{1}\left(\left.\nu\right|_{S}\right)$ of $\left.\nu\right|_{S}$ is given by $(h-k)$-times a generator of $H^{2}(S ; Z)$.

PROOF. For a quaternion number $w$, let indexed letters $w_{1}$ and $w_{2}$ represent the complex numbers such that $w=w_{1}+w_{2} j$.

We note that there exist $S^{1}$-invariant submanifolds of $D_{h, k}(H)$ defined by

$$
\begin{aligned}
& B=\left\{v=0, v^{\prime}=0\right\} \cap D_{h, k}(H), \\
& C=\left\{u_{2}=v_{1}=0, u_{2}^{\prime}=v_{1}^{\prime}=0\right\} \cap D_{h, k}(H),
\end{aligned}
$$

whose intersection is just $S$. (See Figure 1.)

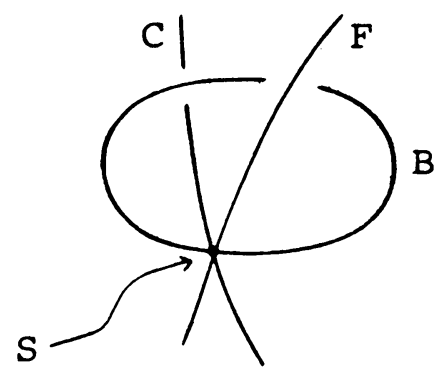

FIGURE 1

This means that $\left.\nu\right|_{S}$ decomposes into the Whitney sum of two complex line bundles $\nu_{B}$ and $\nu_{C}$ where $\nu_{B}$ (resp. $\nu_{C}$ ) is the normal bundle of $S$ in $B$ (resp. $C$ ). As is easily seen, $\nu_{B}$ is trivial and $\nu_{C}$ is isomorphic to the $(h-k)$-times tensor bundle of the canonical complex line bundle over $S$ or to its dual bundle. This proves the lemma. Q.E.D.

Consider the commutative diagram (up to homotopy)

$$
\begin{aligned}
& \partial F \stackrel{\bar{\pi}}{\longrightarrow} S \\
& \kappa \downarrow \quad \lambda / / \pi \pi \\
& F
\end{aligned}
$$

where $\kappa, \lambda$ are the inclusions and $\pi, \bar{\pi}$ are the natural projections. Since the composition $\lambda \circ \pi$ induces the identity map in cohomology, it follows from the naturality of characteristic classes and the (homotopy) commutativity of the above diagram that

$$
c_{1}\left(\left.\nu\right|_{\partial F}\right)=\kappa^{*} c_{1}(\nu)=\kappa^{*} \pi^{*} \lambda^{*} c_{1}(\nu)=\kappa^{*} \pi^{*} c_{1}\left(\left.\nu\right|_{S}\right)=\bar{\pi}^{*} c_{1}\left(\left.\nu\right|_{S}\right) .
$$

Here, since $\bar{\pi}: \partial F \rightarrow S$ is an $S^{1}$-bundle with the Euler class $m$-times a generator of $H^{2}(S ; Z)$, the Thom-Gysin exact sequence of this bundle implies that $\bar{\pi}^{*}: H^{2}(S ; Z) \rightarrow H^{2}(\partial F ; Z) \simeq Z / m Z$ is surjective. This fact, the above identity and Lemma 1.2 show that $c_{1}\left(\left.\nu\right|_{\partial F}\right)$ is given by $(h-k)$-times a generator of $H^{2}(\partial F ; Z)$. Thus we have proved

LEMMA 1.3. If the complex normal bundle of the $S^{1}$-fixed point set in $S_{h, k}(H)$ is trivial, then the difference $h-k$ must be divisible by $m$ where $h+k= \pm m$ as before.

Now suppose that $\Sigma_{m}^{7}$ and $S_{h, k}(H)$ are equivariantly diffeomorphic as $S^{1}$-manifolds. Then Lemmas 1.1 and 1.3 mean that $m$ must divide $h-k$. On the other 
hand, Davis obtained a condition $(h-k)^{2}=|h+k|=m$ by computing their $\mu$ invariants (see p. 83 of [2]). These show $m=1$ and $\{h, k\}=\{ \pm 1,0\}$, which proves the theorem in the case where $\Lambda=H$.

In the case where $\Lambda=O$, we notice that there exists a suitable circle subgroup of $G^{\Lambda}=G_{2}$ such that the fixed point sets in $\Sigma_{m}^{15}$ and $S_{h, k}(O)$ are respectively $\Sigma_{m}^{7}$ and $S_{h, k}(H)$, which still support $S^{1}$-actions (note that the rank of $G_{2}$ is two). Therefore this case reduces to the previous one. The details are left to the reader.

2. Montgomery-Yang correspondence. In this section we review briefly the correspondence discovered by Montgomery and Yang [5] and state a known result concerning it. The reader is referred to [5] for details.

First we introduce an abelian group $\Pi$ : the group of diffeomorphism classes of all homotopy $C P^{3}$ (see p. 484 of $[\mathbf{5}]$ for the sum operation).

Let $\Sigma$ be a representative of an element of $\tilde{C}_{7}(\mathrm{SO}(2))$ (See Introduction for $\left.\tilde{C}_{7}(\mathrm{SO}(2))\right)$. The normal bundle of the $S^{1}$-fixed point set $F\left(S^{1}, \Sigma\right)$ has the complex structure indicated in $\S 1$. We note that this bundle is trivial because $F\left(S^{1}, \Sigma\right)$ is a 3 -sphere and $\pi_{2}(U(2))$ vanishes. This fact ensures the existence of an equivariant embedding

$$
\psi: S^{3} \times \tilde{D}^{4} \rightarrow \Sigma \quad\left(\psi\left(S^{3} \times\{0\}\right)=F\left(S^{1}, \Sigma\right)\right)
$$

such that the $S^{1}$-manifold $\tilde{\Sigma}$ resulting from the equivariant surgery using $\psi$ becomes a homotopy 7 -sphere, where $\tilde{D}^{4}$ stands for the unit 4-disk of $C^{2}$ with the $S^{1}$-action induced from the complex multiplication. Here the $S^{1}$-action on $\tilde{\Sigma}$ is free, and hence the $S^{1}$-orbit space $\tilde{\Sigma} / S^{1}$ is a homotopy $C P^{3}$. The correspondence $\Sigma \rightarrow \tilde{\Sigma} / S^{1}$ defines a map $\rho: \tilde{C}_{7}(\mathrm{SO}(2)) \rightarrow \Pi$, which is the desired one.

Furthermore there is a map $\gamma: \Pi \rightarrow Z$ defined as follows: to $X \in \Pi$ we assign the integer $\gamma(X)$ determined by $p_{1}(X)=(4+24 \gamma(X)) x^{2}$ where $p_{1}(X)$ stands for the first Pontrjagin class of $X$ and $x$ is a generator of $H^{2}(X ; Z)$.

With these understood we have

THEOREM 2.1 [3, 5-7]. The maps $\rho$ and $\gamma$ are both isomorphisms.

REMARK. The isomorphism of $\rho$ is established in [3 and 5], and that of $\gamma$ is due to $[6$ and 7$]$.

3. An alternative proof of Theorem B. Our aim in this section is to give an alternative proof to Theorem B. By Theorem 2.1 it is sufficient to show that the first Pontrjagin class of the homotopy $C P^{3}$ obtained from $S_{2,-1}(H)$ through the correspondence $\rho$ is given by $(4 \pm 24) x^{2}$, where the sign before 24 depends on a choice of orientations of $S_{2,-1}(H)$. This is done in the following.

Let $\psi: S^{3} \times \tilde{D}^{4} \rightarrow S_{2,-1}(H)$ be an equivariant embedding described in $\S 2$. Attach $D_{2,-1}(H)$ and $D^{4} \times \tilde{D}^{4}$ via $\psi$ and denote the resulting $S^{1}$-manifold by $N$. The boundary is a homotopy 7 -sphere $\left(S_{2,-1}(H)\right)^{\sim}$ with a free $S^{1}$-action whose orbit space $\left(S_{2,-1}(H)\right)^{\sim} / S^{1}$ is the desired homotopy $C P^{3}$. Let $L$ be the total space of the 2-disk bundle associated with the $S^{1}$-bundle $\left(S_{2,-1}(H)\right)^{\sim} /\left(S_{2,-1}(H)\right)^{\sim} / S^{1}$. Identifying the boundaries of $N$ and $L$, we obtain a closed, connected and orientable 8-manifold $M$ with a semifree $S^{1}$-action. (See Figure 2.)

Observe that the $S^{1}$-fixed point set consists of two connected components: one of them is diffeomorphic to $C P^{2}$, written $F_{1}$, and the other is $\left(S_{2,-1}(H)\right) \sim / S^{1}$, 
written $F_{2}$. Let $\nu_{i}$ be the complex normal bundle of $F_{i}$ and express their Chern classes as

$$
c\left(\nu_{1}\right)=\left(1+\xi_{1}\right)\left(1+\xi_{2}\right), \quad c\left(\nu_{2}\right)=1+x,
$$

where the former is a formal expression. We remark that $x$ is a generator of $H^{2}\left(F_{2} ; Z\right)$ because it coincides with the Euler class of the $S^{1}$-bundle $\left(S_{2,-1}(H)\right)^{\sim} \rightarrow$ $\left(S_{2,-1}(H)\right)^{\sim} / S^{1}$ and $\left(S_{2,-1}(H)\right)^{\sim}$ is a homotopy 7 -sphere.

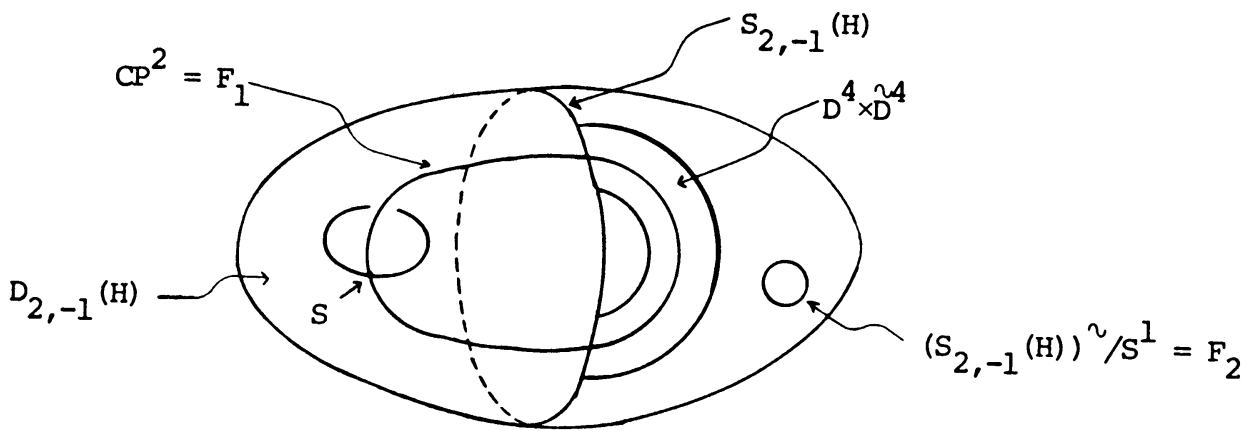

FIGURE 2

Choose an orientation on $M$ arbitrarily and fix it. We take the orientation on $F_{i}$ compatible with the orientation of $M$ and the fiber orientation of $\nu_{i}$ induced from the complex structure. The fundamental class of $F_{i}$ is denoted by $\left[F_{i}\right]$.

With these understood, applying the $G$-signature theorem (see [1]) to our $S^{\mathbf{1}}$ manifold $M$, the signature $\operatorname{Sign} M$ of $M$ is expressed as

$$
\begin{aligned}
\operatorname{Sign} M= & 4\left(1+p_{1}\left(F_{1}\right) / 12\right)\left(\frac{t e^{\xi_{1}}+1}{t e^{\xi_{1}}-1}\right)\left(\frac{t e^{\xi_{2}}+1}{t e^{\xi_{2}}-1}\right)\left[F_{1}\right] \\
& +8\left(1+p_{1}\left(F_{2}\right) / 12\right)\left(\frac{t e^{x}+1}{t e^{x}-1}\right)\left[F_{2}\right],
\end{aligned}
$$

where $t$ is the standard complex one dimensional $S^{1}$-module.

We shall compute the right-hand side of (3.2). We first notice that $p_{1}\left(F_{1}\right)=3 \alpha^{2}$ as $F_{1}$ is diffeomorphic to $C P^{2}$ where $\alpha$ is a generator of $H^{2}\left(F_{1} ; Z\right)$.

On the other hand, an elementary calculation yields

$$
\begin{aligned}
\frac{t e^{y}+1}{t e^{y}-1}=\frac{t+1}{t-1}\left\{t+\frac{2 t}{1-t^{2}} y+\frac{t}{(t-1)^{2}} y^{2}-\frac{t\left(t^{2}+4 t+1\right)}{3(t-1)^{3}(t+1)} y^{3}\right. & + \text { the higher terms in } y\} .
\end{aligned}
$$

Using this expansion together with $p_{1}\left(F_{1}\right)=3 \alpha^{2}$ for the right-hand side of (3.2), it reduces to

$$
\begin{aligned}
\operatorname{Sign} M= & \frac{1}{(t-1)^{2}}\left\{(t+1)^{2} \varepsilon-4 t x p_{1}\left(F_{2}\right)\left[F_{2}\right] / 3\right\} \\
& +\frac{4 t}{(t-1)^{4}}\left\{4 t e+(t+1)^{2} p-2\left(t^{2}+4 t+1\right) x^{3}\left[F_{2}\right] / 3\right\},
\end{aligned}
$$

where $p=p_{1}\left(\nu_{1}\right)\left[F_{1}\right], e=e\left(\nu_{1}\right)\left[F_{1}\right](e(\cdot)$ stands for the Euler class $)$ and $\varepsilon=\alpha^{2}\left[F_{1}\right]$. We remark that $\varepsilon$ is equal to \pm 1 as $\alpha$ is a generator of $H^{2}\left(F_{1} ; Z\right)$. 
We know that $\operatorname{Sign} M$ is a constant integer, so there must be no pole at $t=1$ on the right-hand side of (3.3). This fact imposes the following condition:

$$
\left(x p_{1}\left(F_{2}\right)-4 x^{3}\right)\left[F_{2}\right]=-3(p+2 e-\varepsilon) .
$$

Now consider the restriction of $\nu_{1}$ to a 2 -sphere $S$ defined in $\S 1$. Since the inclusion from $S$ to $F_{1}$ induces an isomorphism of cohomology groups in degree two, Lemma 1.2 means that $c_{1}\left(\nu_{1}\right)=3 \alpha$ up to sign. This and the general relation $p_{1}(\cdot)=c_{1}(\cdot)^{2}-2 c_{2}(\cdot)=c_{1}(\cdot)^{2}-2 e(\cdot)$ deduce $p+2 e=9 \varepsilon$. Thus it follows from this identity and (3.4) that

$$
\left(x p_{1}\left(F_{2}\right)-4 x^{3}\right)\left[F_{2}\right]=-24 \varepsilon
$$

which proves Theorem $\mathrm{B}$ as $\varepsilon= \pm 1$.

\section{REFERENCES}

1. M. F. Atiyah and I. M. Singer, The index of elliptic operators. III, Ann. of Math. (2) 87 (1968), 546-604.

2. M. Davis, Some actions on homotopy spheres of dimension seven and fifteen, Amer. J. Math. 104 (1982), 59-90.

3. J. Levine, Semi-free circle actions on spheres, Invent. Math. 22 (1973), 161-186.

4. M. Masuda, Smooth involutions on homotopy $C P^{3}$, Amer. J. Math (to appear).

5. D. Montgomery and C. T. Yang, Differentiable actions on homotopy seven spheres, Trans. Amer. Math. Soc. 122 (1966), 480-498.

6. __ Differentiable actions on homotopy seven spheres. II, Proc. Conf. Transformation Groups (New Orleans 1967), Springer, New York, 1968, pp. 125-134.

7. C. T. C. Wall, Classification problems in differential topology. V, On certain 6-manifolds, Invent. Math. 1 (1966), 355-374.

Department of Mathematics, Faculty of Science, University of Tokyo, HONGO, TOKYO 113, JAPAN 